\title{
Automated tracking systems for the assessment of farmed poultry
}

\author{
Suresh Neethirajan ${ }^{1^{*}}$ \\ ${ }^{1}$ Adaptation Physiology Group, Department of Animal Sciences, Wageningen University \& \\ Research, 6700 AH Wageningen, The Netherlands \\ * Correspondence: suresh.neethirajan@wur.nl
}

\begin{abstract}
The world's growing population is highly dependent on animal agriculture. Animal products provide nutrient-packed meals that help to sustain individuals of all ages in communities across the globe. As the human demand for animal proteins grows, the agricultural industry must continue to advance its efficiency and quality of production. One of the most commonly farmed livestock is poultry and their significance is felt on a global scale. Current poultry farming practices result in the premature death and rejection of billions of chickens on an annual basis before they are processed for meat. This loss of life is concerning regarding animal welfare, agricultural efficiency, and economic impacts. The best way to prevent these losses is through the individualistic and/or group level assessment of animal on a continuous basis. On large-scale farms, such attention to detail was generally considered to be inaccurate and inefficient, but with the integration of Artificial Intelligence (AI) assisted technology individualized and per-herd assessments of livestock are possible and accurate. Various studies have shown cameras linked with specialized systems of AI can properly analyze flocks for health concerns, thus improving the survival rate and product quality of farmed poultry. Building on the recent advancements, this review explores the aspects of AI in the detection, counting and tracking of the poultry in commercial and research-based applications.
\end{abstract}

Keywords: Poultry behaviour; target tracking; deep learning; precision livestock farming; poultry production systems.

\section{Introduction}

Today's demands for increased livestock production result in various challenges for the animals they pertain to. A balance is needed between the quantity and quality of poultry production. However, farmers must worry about maximizing profits, a need that has promoted a prioritization of production over aspects like welfare. Flock size and growth are commonly maximized in minimal spaces to offset low margins for farmers. Societal pressures towards sustainability also influence minimal inputs for poultry farming aspects like land, labor, and natural resource usage. These efforts may lead to increased poultry production with decreased production time and resource usage, but they have also unintentionally led to the proliferation of harmful genetic alterations and the increase of associated diseases. The solution to these complex agricultural needs is to assist farmers with automated surveillance of the animals. Through the continuous and automated monitoring of animals, farmers are able to detect welfare and production concerns in a manner that is both quick and reliable. With the integration of modern technological advances, poultry farming has the opportunity to grow in terms of production quantity and animal care quality with minimal added expense. 
On a global scale, 60 billion chickens are raised for meat production every year, but not all of them make it to people's plates. Three billion of these chickens don't survive the rearing process, and 1.6 billion are rejected at the slaughterhouse because of illnesses, scratches, bruises, and other signs of welfare failures. Next to 60 billion, 4.6 billion may sound like a small figure, but it is the difference between food accessibility and hunger for some people, and for farmers, it is a great source of profit loss. This statistic also makes a huge difference for the animals, as it suggests that 4.6 billion chickens bred for meat suffer from unmanaged, painful, and possibly deadly medical (pathological) conditions each year. An inefficiency in feed to production ratios can also be noted in this statistic, as the 3 million tons of feed used to grow the 4.6 billion chickens that are discarded every year. With better diagnostics and agricultural management, fewer resources would be wasted, more chickens could be produced, and less suffering would be faced by these animals.

There is a need to increase agricultural capabilities to detect anomalies in chicken behavior and health and thereby welfare without increasing a need for manual labor, and for that, automated systems are needed. Automated systems have been studied and proven to be capable of accurately collecting data related to the following needs.

- Individual tracking, even in large groups of animals that are condensed in a confined space.

- Phenotype assessment and analysis for the non-invasive understanding of genotypes, which are important for resilient breeding methods.

- Identification of the needs of individual animals in relation to welfare.

- Continuous data collecting capabilities that cannot be replicated by humans.

- $\quad$ Assessment of activity and changes on a flock level.

- $\quad$ Early direction of behavioral and physical shifts in comparison to past flocks.

- Analysis of nuances related to welfare-focused farming, like the preferences in light intensity for individuals or groups of agricultural birds.

- $\quad$ Long-range use for the non-disruptive observation of fearful and free-range livestock.

- Bone fracture assessment for immediate intervention

\section{Need for automated poultry surveillance}

Poultry and eggs are a major source of dietary protein for people across the globe (Lin et al., 2020). As a result, these animal food sources must be produced in a way that minimizes their cost and maximizes their availability if poultry is going to remain a major food source as the human population continues to grow. The profitability and productivity of commercial poultry farming depend on regular monitoring of the birds, and minimal human labor to maintain its affordability (Okinda et al., 2020). The modern solution to this issue can be found in technological advances that are both growing in accuracy and decreasing in price. Introducing artificial intelligence in poultry farming and management has the potential to improve multiple aspects of the industry. With the ability to accumulate data that triggers informed actions, this technology has the potential to improve animal welfare, minimize the spread of disease, improve breeding standards, and reduce waste (Gebhardt-Henrich et al., 2021). With so many promising implications, it should be no surprise that automated poultry surveillance is receiving plenty of attention in the realm of research. 
Many of the production economics of poultry farms depend upon visually accessible aspects, such as the size, weight and appearance of poultry eggs and meat. This is precisely why the computerized video-based system is becoming a popular real-time automated tool for poultry processing. It is praised as a non-intrusive and non-invasive option for flock assessment that seriously reduces and even eliminates events of unnecessary stress that are commonly caused by human observation. This aspect makes it a beneficial tool for presenting a wide range of data on animals within a flock and for the sorting and grading of poultry-related products (Nyalala et al., 2021).

The detection and prediction of abnormal behavior and poultry diseases can be accurately managed using automated tracking platforms (Fang et al., 2020). These systems are capable of recording data and analyzing poultry farming focuses, including;

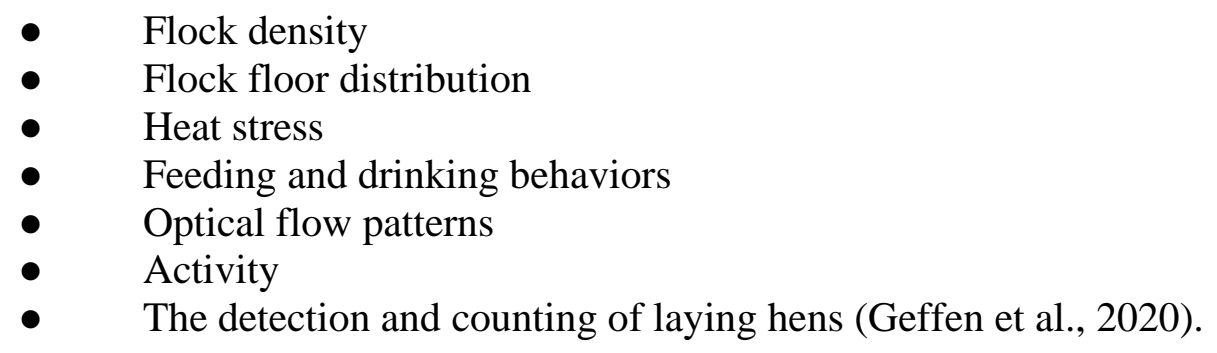

This technology also permits the use of robust detection of eggs, which will make the tedious and time-consuming task of floor egg collection easier for farmers (Li et al., 2020a). The behavioral issues in group-housed turkeys, such as cannibalism, can be rapidly detected and consequently addressed through deep learning techniques (Nasirahmadi et al., 2020). Some systems were even developed to find the location of chickens on a farm for simplified assessment and treatment by farmers (Rufener et al., 2018).

\section{Artificial intelligence in poultry monitoring}

Currently, the role of artificial intelligence in various aspects of society is becoming increasingly obvious to the public, so it is no surprise that this method of management is making its way into food production systems. Computerized monitoring technology promises to fulfill the growing criteria for improved poultry production management, including conversion of the feed ratios and profitability (Natasha et al., 2020).

\section{Computer vision technology}

The welfare factors related to farm management can be better understood by monitoring poultries' natural processes and responses. Computer video systems can assess and determine a wide range of data at a time, including housing management, weight measurement, behaviour, detection of diseases, slaughtering processes, eggs quality, and carcass quality checking (Okinda et al., 2020) (Natasha et al., 2020).

\section{Basic parts}


The computer tracking and monitoring technology for various poultry processes consists of two main parts (Natasha et al., 2020). The first of which is the hardware. Hardware is recognized as the physical components of these systems, which include wiring and other tangible components. Advancements in hardware are the primary reason for the development of vision technology in poultry farming. There are three key components of functional hardware in computer vision systems.

- Cameras and various lens suited to the environment and assigned task

- Lighting units

- Mounts that allow for the full view of an observed farming space without interrupting normal poultry functions.

The second component is software. This includes the programs and other operating information needed for the hardware to perform its specified function. Software is specially designed for data acquisition and data analysis, especially in the field of agriculture, where it must be altered to suit the species of interest. Data acquisition software system performs its role in the storage and selection of good quality images (or videos) that are produced by the cameras. Data analytical platforms helps in the processing of images using algorithms suited to the data and research needs (Natasha et al., 2020).

\section{Types}

Computerized visual analysis systems exist in two major forms, which are identified as Machine learning-based systems and deep learning-based systems.

\section{Machine Learning-based Systems}

Machine learning-based computer vision systems follow a specific image analysis protocols and a specially designed algorithm. The basic workflow of a machine learning-based system for poultry monitoring is as follows (Okinda et al., 2020);

- $\quad$ Acquisition of image: Focused on depth or RGB images.

- $\quad$ Pre-processing of image: Normalization, resizing, color-space transformation

- ROI (Region of interest) Segmentation: Background removal or subtraction, ellipse modeling, and other focus enhancing alterations

- $\quad$ Features extraction: Optical flow meter, locomotor and morphological features

- $\quad$ Modeling: Machine learning-based algorithms

- $\quad$ Regression: Monitoring of bioprocess and bio responses

\section{Deep Learning-based systems}

The deep learning-based computer vision system is the recent advancement in the automatic livestock observation, and it simplifies associated data processing. Various processes of machine learning systems like segmentation, feature extraction, and selection are time consuming and subjective laborious tasks. It is also important to note that the performance of these algorithms must change in relation to sensor sensitivity (Okinda et al., 2020). The most important feature of a deep learning system is its ability to directly process the image, thus eliminating older laborious 
processes by using Deep Neural Net (DNN). These deep learning models generally provide higher accuracy than machine learning, making them better suited to the observation of large flocks (Okinda et al., 2020). Deep learning systems also solve the common complications with multiple objects tracking when using a single camera. This is a revolutionary advancement for researchers and farmers since it minimizes equipment costs (Ciaparrone et al., 2020).

\section{Applications}

Computer vision systems can be adapted to suit a variety of applications, including the following, which are geared towards poultry farming (Okinda et al., 2020).

- $\quad$ Recognition and identification of images: Checking for the presence of poultry in every image.

- $\quad$ Detection of object: Locating the exact position of poultry in every image.

- Classification of image: Classifying the identified poultry as sick or absent.

- Segmentation: Identifying the watering and feeding structures in every image.

- Recognition of specific objects: Noting the behaviors exhibited by members of a flock.

\section{Milestones in the field of automated poultry tracking}

Different techniques and methods have been developed in the poultry industry to ensure improved production rates (Ichiura et al., 2019). Among the poultry species farmed globally, chickens are the most common and produced in the largest quantities.

\section{Individual Chicken Identification in Crowded Free-Ranging Spaces}

The individual tracking of poultry has the benefit of evaluating abnormal behavior and predicting diseases for immediate treatment, but this can be a near-impossible task in crowded housing situations (Sibanda et al., 2020). For this purpose, scientists devised a system to individually track a bird by using image thresholding, feature engineering, and morphological transformation (Novas and Usberti, 2017).

The non-invasive integration of a deep regression network has the capacity to greatly enhance the functionality of poultry farms down to the individual level. Various comparative research studies have proven that TBroiler, a poultry tracking algorithm, shows superior results based on pixel error, failure rate and overlap ratio when compared to older algorithms (Fang et al., 2020). Furthermore, researchers have made the system check the relationship between spatial memory and the ranging behavior of free-range broiler chickens for more in-depth analysis (Ferreira et al., 2019). This method of visual identification is also effective in locating free-range layer eggs with great accuracy so robotic collection methods can function efficiently ( $\mathrm{Li}$ et al., 2020).

\section{Detection of Broiler Movements Through Optical Flow Patterns}

The technological advancement of poultry farms comes with extraordinary animal welfare and productivity improvements on both the individual and flock scale (Yang et al., 2020). These advancements have also made it possible for researchers to conduct life-long monitoring of individual birds or entire flocks as needed (Dawkins et al., 2012). 
Gait variation between broiler chickens is a tell-tale sign of abnormalities in physical conformation and developmental complications. Normally a human would be tasked with observing and rating the gaits of broiler chickens. However, that task consumes a great amount of time and money despite its inaccuracies from human biases and unintentional influences. Automated machine vision cameras can be specially programmed to detect any variation in gait among individuals in a flock at speeds and consistencies that reach far beyond those of a human. Through optical flow patterns, variations in the gaits of slow-moving birds are easily identifiable on an individual level, especially in comparison to fast-moving birds that display a uniform motion of individuals (Gebhardt-Henrich et al., 2021). This form of information collection is crucial for the non-invasive analysis of bone strength, keel health, and bone structure in poultry since it does not require stressful human interactions.

This form of tracking also opens up the opportunity to include 3D camera technology. These cameras use a depth sensor to identify the lameness of individual broiler chickens through body positioning and inactivity that can only be assessed using depth perception (Aydin, 2017b). Another technique for assessing lameness in broiler chickens includes the use of an image analysis algorithm that detects motion variables like lateral body oscillation, speed, step length, and step frequency (Aydin, 2017a).

\section{Increasing poultry productivity through time series data mining}

Advancements in sensor technologies make it easy for farmers to record and measure animal behavior patterns and provide timely interventions or decision-making capabilities. However, there still are challenges that must be faced to achieve a booming production poultry rate (Abdoli et al., 2020).

Complications like heat stress are unavoidable in some areas, and the increases in temperatures can cause natural behavioral changes in poultry. Most commonly, heat stress causes broiler chickens to reduce activity which can skew typical observation results towards inaccurate rates of lameness. Luckily, researchers have proposed a model for recognizing heat stress by making use of the YOLOv3 algorithm. This algorithm increases the accuracy of lameness assessments in flocks to a satisfactory rate of $83 \%$ (Anlan et al., 2019).

\section{Image analysis of broiler chicken behavior at different feeders}

The feeding patterns of poultry, like broiler chickens, dictates the type of feeders needed for proper nutrition and behavioral control (Li et al., 2020b). In one study, three types of feeders were used. These feeders included automatic systems with a partition grid, tubing systems, and tube systems without a barrier grid. Observations were then recorded using computational image data analysis. The activity index was a variable not influenced by the feeder type, but other behavioral variables showed some relation. The conclusion of this study showed that the design of poultry feeders has significant impacts and that feeding behaviors can be accurately monitored through automated image analysis (Neves et al., 2015).

\section{Detection of poultry diseases using Deep Learning system and image analysis}


Diseases in confined poultry farming operations spread quickly, leading to welfare issues for the animals and significant financial losses for farmers (Zhuang and Zhang, 2019). In a majority of situations, diseases are not detectable by manual observation. However, there are certain observable factors that signify a healthy flock, such as the spatial distribution of animals (Guo et al., 2020).

Flock attributes like spatial distribution are best detected through visual observations. This leads to the use of digital image analysis and deep learning systems. In one study of a modernized system, an Improved Feature Fusion Single Shot MultiBox Detector (IFSSD) along with the single shot multibox detector was proposed as a method of enhanced algorithmic assessment. The IFSSD can improve and enrich the image quality, making it easy to detect sick broiler chickens amongst large flocks (Zhuang and Zhang, 2019).

Through usage of optical flow, one can easily detect visual ailments like dermatitis and hock burn in poultry (Dawkins et al., 2017). Another milestone in modern poultry farming is installing a system to detect Campylobacter-positive birds using cameras and analyzing optical flow patterns. The detection of high movement peaks and lower mean optical flow patterns indicate that a flock could be carriers of Campylobacter - a major cause of gastrointestinal infections in humans (Colles et al., 2016).

Zoonotic and highly contagious diseases, like bird flu, cause economic losses to farmers and threaten human health (Zhuang et al., 2018). Historically, such diseases are not recognized and treated until after they have done significant damage, but the use of real-time image monitoring provides a simple and effective solution (Zhuang et al., 2018). Healthy and sick broiler chickens can be easily differentiated through posture comparisons, allowing farmers to take immediate outbreak-preventing actions.

\section{Infrared receiver assessments of keel bone fractures in laying hens}

The keel is a crucial physiological structure in chickens that is closely related to locomotion. It can be damaged in a number of ways, leading to lameness and even premature death. To better assess the occurrences of keel bone fractures, researchers created a real-time tool for the assessment of laying hens by using infrared receivers. Here, they attached infrared receivers to the legs of the hens and monitored their behavior patterns (Rufener et al., 2019). This minimally invasive method of measuring lameness and keel health is practical and effective for the assessment of small flocks, but it is less practical for use in larger poultry farming operations. Per-animal approach in poultry is practically not possible unlike the cattle and swine industry. Passive radio frequency systems function in a similar method to these infrared receivers and provide clear, individualized points of information on the animals within a flock, but they face the same issue of impracticality on large scales (van der Sluis et al., 2020).

\section{Evaluation of laying hens' light preferences}

Researchers developed a visual-based light preference test system to detect and count laying hens. Light may seem like a minor aspect of farming, but it can have a significant impact on the laying 
quantities and life quality of laying hens. In this study, two algorithms were used, one for the analysis of images and the other for weight. The accuracy of this test system was based on automatic visual observation, which made it possible to detect the laying hens in each light compartment along with their number (Li et al., 2018).

An automated system was designed to detect the laying hens present in battery cages by using cameras along with the tracking algorithms and automated video technology for the detection of laying hens occupying multiple nests (Geffen et al., 2020) (Zaninelli et al., 2018). This technology has also served to assess the range of individual layers for better production management. It works by using radio-frequency technology that tags laying hens individually (Campbell et al., 2018). This method is practical only in larger livestock or research animals, as poultry are generally farmed in large quantities that would require time and large financial investments for the individual tagging of an entire flock.

\section{Deep Learning system detection of pecking activity in grouped-housed turkeys}

When Turkeys are raised in artificially confined environments, they become more prone to cannibalistic behaviors that can be distinguished through pecking and movement patterns. These behaviors must be caught early and bred out of a gene pool to avoid catastrophic financial losses and unnecessary animal suffering. Two metallic balls have been used along with a microphone to record pecking audios that suggest behavioral changes. Video data was also recorded by a camera mounted for a top view of the study subjects. Using the data from these two sources, cannibalistic pecking activity measurement in turkeys has been demonstrated with convolutional neural network (CNN) models for a full assessment and intervention related to this destructive behavior (Nasirahmadi et al., 2020).

\section{Tracking and stocking density estimation}

The monitoring of individual (per-animal) poultry birds is a difficult task, even with the use of video-based monitoring applications. The similar appearances of individuals, occlusions, and other technical complications motivated scientists to develop a robust detection, counting and tracking method capable of assessing multiple animals at once. The primary feature of this observation method is its ability to monitor the animals constantly. It can even collect data at night by using infrared cameras through heat map-based classification and evaluation. This technological advancement allows farmers to measure and estimate stock density and behavioral patterns on an individual basis (Zhang et al., 2019). Deep and shallow vision technologies can also be implemented in near future to help assess behavioral pattern changes such as pecking over time (Bergamini et al., 2021).

Table 1: An overview of current research advancements of automated poultry monitoring tools

\begin{tabular}{lll}
\hline Applications & $\begin{array}{l}\text { Used Tools \& } \\
\text { Platforms }\end{array}$ & $\begin{array}{l}\text { Solved Poultry References } \\
\text { Problems }\end{array}$ \\
\hline
\end{tabular}




\begin{tabular}{llll}
\hline Counting of individual broiler & Camera, TBroiler & $\begin{array}{l}\text { Abnormal } \\
\text { behavior; patterns }\end{array}$ & (Fang et al., 2020 \\
\hline Broiler movement & Camera & $\begin{array}{l}\text { Variations among } \\
\text { individuals }\end{array}$ & $\begin{array}{l}\text { (Gebhardt- Henrich et al., } \\
\text { 2021). }\end{array}$
\end{tabular}

\begin{tabular}{|c|c|c|c|}
\hline Productivity in broiler & Camera, sensors & $\begin{array}{l}\text { Advance } \\
\text { treatments for } \\
\text { healthy growth }\end{array}$ & $\begin{array}{l}\text { (Abdoli et al., } \\
\text { 2020). }\end{array}$ \\
\hline Behavior at different feeders & Camera & $\begin{array}{l}\text { Choice of feeder } \\
\text { design }\end{array}$ & $\begin{array}{l}\text { (Neves } \\
2015)\end{array}$ \\
\hline Detection of disease & Camera, IFSSD & $\begin{array}{l}\text { Outbreak } \\
\text { prevention }\end{array}$ & $\begin{array}{l}\text { (Dawkins et al., } \\
\text { 2017). }\end{array}$ \\
\hline Sick broiler assessment & Camera & $\begin{array}{l}\text { Disease } \\
\text { management }\end{array}$ & $\begin{array}{l}\text { (Zhuang et al., } \\
\text { 2018). }\end{array}$ \\
\hline Keel bone fracture & Infrared receivers & Timely treatments & $\begin{array}{l}\text { (Rufener et al., } \\
\text { 2019). }\end{array}$ \\
\hline
\end{tabular}

\begin{tabular}{llll}
\hline Laying hen light preference & $\begin{array}{l}\text { Camera, tracking } \\
\text { algorithm }\end{array}$ & $\begin{array}{l}\text { Layer detection in (Li et al., 2018) } \\
\text { cages }\end{array}$ \\
\hline Pecking in turkeys & $\begin{array}{l}\text { Camera, } \\
\text { microphone, and } \\
\text { metallic balls }\end{array}$ & $\begin{array}{l}\text { Assessment of } \\
\text { cannibalism }\end{array}$ & $\begin{array}{l}\text { (Nasirahmadi et 2020). } \\
\text { al. }\end{array}$ \\
\hline Tracking in pigs & Camera, sensors & $\begin{array}{l}\text { Individual } \\
\text { behavior }\end{array}$ & $\begin{array}{l}\text { (Zhang et al., } \\
\text { 2019) }\end{array}$ \\
\hline
\end{tabular}




\section{Future Outlook}

Continuous real-time heterogeneous data streaming from multiplexed sensors in the poultry barn for automated decision-making processes is still a challenge. Due to advancements in the CNN, hardware manufacturers have introduced high throughput internet data transfer capabilities of over 10 Tera operations per second. However, currently most of the automated detection and tracking systems in poultry industry are essentially passive and cannot control or interface with changing the ventilation systems or feed inlet controls or creating alarms or call-for-actions to veterinarians. The automated tracking of poultry platform requires the detection, selection and tracking of activity of the poultry without the birds having any prior stored trajectory (motion planning prediction). This is a bottleneck in the image processing aspects, but AI based prediction algorithms may be able to overcome this barrier. Further challenges in practical realization of the automated systems involves multiplexing of sensor technologies such as ultrasonic, LIDAR, cameras and real-time data processing hardware in achieving the accuracy and thereby the prediction of the poultry movement and behavior. The use of AI enabled technology in poultry farming is essential for increasing the production rate and improve welfare-based farming practices. Economic losses and suffering can be effectively prevented through the automated visual detection of diseases. These advancements in individual and flock behavioral evaluations will help the poultry agricultural sector grow in production without the need for major sacrifices along the way.

\section{References}

1. Abd Aziz, N. S. N., Mohd Daud, S., Dziyauddin, R. A., Adam, M. Z., \& Azizan, A. (2021). A Review on Computer Vision Technology for Monitoring Poultry Farm-Application, Hardware, and Software. IEEE Access, 9, 12431-12445. https://doi.org/10.1109/ACCESS.2020.3047818

2. Abdoli, A., Alaee, S., Imani, S., Murillo, A., Gerry, A., Hickle, L., \& Keogh, E. (2020, August 22). Fitbit for Chickens? Time Series Data Mining Can Increase the Productivity of Poultry Farms. https://doi.org/10.1145/3394486.3403385

3. Anlan, D., Xuhui, Z., Xiu-guo, Z., Yan, Q., Heyang, Y., Shi-kai, Z., \& Yu-ning, W. (2019). A novel method for the group characteristics analysis of yellow feather broilers under the heat stress based on object detection and transfer learning. https://doi.org/10.35633/inmateh-59-06

4. Aydin, A. (2017a). Using 3D vision camera system to automatically assess the level of inactivity in broiler chickens. Computers and Electronics in Agriculture, 135, 4-10. https://doi.org/10.1016/j.compag.2017.01.024

5. Aydin, A. (2017b). Development of an early detection system for lameness of broilers using computer vision. Computers and Electronics in Agriculture, 136, 140-146. https://doi.org/10.1016/j.compag.2017.02.019

6. Bergamini, L., Pini, S., Simoni, A., Vezzani, R., Calderara, S., D’Eath, R., \& Fisher, R. (2021). Extracting Accurate Long-term Behavior Changes from a Large Pig Dataset: Proceedings of the 16th International Joint Conference on Computer Vision, Imaging and Computer Graphics Theory and Applications, 524-533. https://doi.org/10.5220/0010288405240533

7. Campbell, D. L. M., Horton, B. J., \& Hinch, G. N. (2018). Using Radio-Frequency Identification Technology to Measure Synchronised Ranging of Free-Range Laying Hens. Animals, 8(11), 210. https://doi.org/10.3390/ani8110210 
8. Ciaparrone, G., Sánchez, F. L., Tabik, S., Troiano, L., Tagliaferri, R., \& Herrera, F. (2020). Deep Learning in Video Multi-Object Tracking: A Survey. Neurocomputing, 381, 61-88. https://doi.org/10.1016/j.neucom.2019.11.023

9. Colles, F. M., Cain, R. J., Nickson, T., Smith, A. L., Roberts, S. J., Maiden, M. C. J., Lunn, D., \& Dawkins, M. S. (2016). Monitoring chicken flock behaviour provides early warning of infection by human pathogen Campylobacter. Proceedings of the Royal Society B: Biological Sciences, 283(1822), 20152323. https://doi.org/10.1098/rspb.2015.2323

10. Dawkins, M. S., Roberts, S. J., Cain, R. J., Nickson, T., \& Donnelly, C. A. (2017). Early warning of footpad dermatitis and hockburn in broiler chicken flocks using optical flow, bodyweight and water consumption. The Veterinary Record, 180(20), 499. https://doi.org/10.1136/vr.104066

11. Dawkins, Marian Stamp, Cain, R., \& Roberts, S. J. (2012). Optical flow, flock behaviour and chicken welfare. Animal Behaviour, 84(1), 219-223. https://doi.org/10.1016/j.anbehav.2012.04.036

12. Fang, C., Huang, J., Cuan, K., Zhuang, X., \& Zhang, T. (2020). Comparative study on poultry target tracking algorithms based on a deep regression network. Biosystems Engineering, 190, 176-183. https://doi.org/10.1016/j.biosystemseng.2019.12.002

13. Ferreira, V. H. B., Peuteman, B., Lormant, F., Valenchon, M., Germain, K., Brachet, M., Leterrier, C., Lansade, L., Calandreau, L., \& Guesdon, V. (2019). Relationship between ranging behavior and spatial memory of free-range chickens. Behavioural Processes, 166, 103888. https://doi.org/10.1016/j.beproc.2019.103888

14. Geffen, O., Yitzhaky, Y., Barchilon, N., Druyan, S., \& Halachmi, I. (2020). A machine vision system to detect and count laying hens in battery cages. Animal, 14(12), 2628-2634. https://doi.org/10.1017/S1751731120001676

15. Guo, Y., Chai, L., Aggrey, S. E., Oladeinde, A., Johnson, J., \& Zock, G. (2020). A Machine Vision-Based Method for Monitoring Broiler Chicken Floor Distribution. Sensors, 20(11), 3179. https://doi.org/10.3390/s20113179

16. Ichiura, S., Mori, T., Horiguchi, K., \& Katahira, M. (2019). EXPLORING IOT BASED BROILER CHICKEN MANAGEMENT TECHNOLOGY. 7.

17. Lee, D.-H., Kim, A.-K., Choi, C.-H., \& Kim, Y.-J. (2019). Study on image-based flock density evaluation of broiler chicks. The Journal of Korea Institute of Information, Electronics, and Communication Technology, 12(4), 373-379. https://doi.org/10.17661/jkiiect.2019.12.4.373 18. Li, G., Hui, X., Chen, Z., Chesser, G. D., \& Zhao, Y. (2021). Development and evaluation of a method to detect broilers continuously walking around feeder as an indication of restricted feeding behaviors. Computers and Electronics in Agriculture, 181, 105982. https://doi.org/10.1016/j.compag.2020.105982

19. Li, G., Li, B., Shi, Z., Zhao, Y., \& Ma, H. (2018). Design and evaluation of a lighting preference test system for laying hens. Comput. Electron. Agric. https://doi.org/10.1016/j.compag.2018.01.024

20. Li, G., Xu, Y., Zhao, Y., Du, Q., \& Huang, Y. (2020). Evaluating Convolutional Neural Networks for Cage-Free Floor Egg Detection. Sensors, 20(2), 332. https://doi.org/10.3390/s20020332

21. Li, G., Zhao, Y., Purswell, J. L., Du, Q., Chesser, G. D., \& Lowe, J. W. (2020). Analysis of feeding and drinking behaviors of group-reared broilers via image processing. Computers and Electronics in Agriculture, 175, 105596. https://doi.org/10.1016/j.compag.2020.105596 
22. Lin, C.-Y., Hsieh, K.-W., \& Tsai, Y.-C. (2020). Automatic Monitoring of Chicken Movement and Drinking Time Using Convolutional Neural Networks. https://doi.org/10.13031/trans.13607

23. Nasirahmadi, A., Gonzalez, J., Sturm, B., Hensel, O., \& Knierim, U. (2020). Pecking activity detection in group-housed turkeys using acoustic data and a deep learning technique. Biosystems Engineering, 194, 40-48. https://doi.org/10.1016/j.biosystemseng.2020.03.015

24. Neves, D., Mehdizadeh, S., Tscharke, M., Nääs, I., \& Banhazi, T. (2015). Detection of flock movement and behaviour of broiler chickens at different feeders using image analysis. Information Processing in Agriculture, 2. https://doi.org/10.1016/j.inpa.2015.08.002

25. Neves, D. P., Mehdizadeh, S. A., Tscharke, M., Nääs, I. de A., \& Banhazi, T. M. (2015). Detection of flock movement and behaviour of broiler chickens at different feeders using image analysis. Information Processing in Agriculture, 2(3), 177-182. https://doi.org/10.1016/j.inpa.2015.08.002

26. Novas, R. V., \& Usberti, F. L. (2017). Live Monitoring in Poultry Houses: A Broiler Detection Approach. 2017 30th SIBGRAPI Conference on Graphics, Patterns and Images (SIBGRAPI), 216-222. https://doi.org/10.1109/SIBGRAPI.2017.35

27. Nyalala, I., Okinda, C., Kunjie, C., Korohou, T., Nyalala, L., \& Chao, Q. (2021). Weight and volume estimation of poultry and products based on computer vision systems: A review. Poultry Science, 100(5), 101072. https://doi.org/10.1016/j.psj.2021.101072

28. Okinda, C., Nyalala, I., \& Korohou, T. (2020). A review on computer vision systems in monitoring of poultry: A welfare perspective-ScienceDirect. https://www.sciencedirect.com/science/article/pii/S2589721720300258

29. Rufener, C., Berezowski, J., Maximiano Sousa, F., Abreu, Y., Asher, L., \& Toscano, M. J. (2018). Finding hens in a haystack: Consistency of movement patterns within and across individual laying hens maintained in large groups. Scientific Reports, 8(1), 12303. https://doi.org/10.1038/s41598-018-29962-x

30. Rufener, Christina, Abreu, Y., Asher, L., Berezowski, J. A., Maximiano Sousa, F., Stratmann, A., \& Toscano, M. J. (2019). Keel bone fractures are associated with individual mobility of laying hens in an aviary system. Applied Animal Behaviour Science, 217, 48-56. https://doi.org/10.1016/j.applanim.2019.05.007

31. Sibanda, T. Z., Welch, M., Schneider, D., Kolakshyapati, M., \& Ruhnke, I. (2020). Characterising Free-Range Layer Flocks Using Unsupervised Cluster Analysis. Animals : An Open Access Journal from MDPI, 10(5). https://doi.org/10.3390/ani10050855

32. van der Sluis, M., de Haas, Y., de Klerk, B., Rodenburg, T. B., \& Ellen, E. D. (2020). Assessing the Activity of Individual Group-Housed Broilers Throughout Life Using a Passive Radio Frequency Identification System-A Validation Study. Sensors, 20(13), 3612. https://doi.org/10.3390/s20133612

33. Yang, Q., \& Xiao, D. (2020). A review of video-based pig behavior recognition. Applied Animal Behaviour Science, 233, 105146. https://doi.org/10.1016/j.applanim.2020.105146

34. Yang, X., Zhao, Y., \& Tabler, G. T. (2020). Accuracy of Broiler Activity Index as Affected by Sampling Time Interval. Animals, 10(6), 1102. https://doi.org/10.3390/ani10061102

35. Zaninelli, M., Redaelli, V., Luzi, F., Mitchell, M., Bontempo, V., Cattaneo, D., Dell'Orto, V., \& Savoini, G. (2018). Development of a Machine Vision Method for the Monitoring of Laying Hens and Detection of Multiple Nest Occupations. Sensors (Basel, Switzerland), 18(1). https://doi.org/10.3390/s18010132 
36. Zhang, L., Gray, H., Ye, X., Collins, L., \& Allinson, N. (2019). Automatic Individual Pig Detection and Tracking in Pig Farms. Sensors, 19(5), 1188. https://doi.org/10.3390/s19051188 37. Zheng, H., Zhang, T., \& Fang, C. (2021). Animals |Design and Implementation of Poultry Farming Information Management System Based on Cloud Database. https://www.mdpi.com/2076-2615/11/3/900

38. Zhuang, X., Bi, M., Guo, J., Wu, S., \& Zhang, T. (2018). Development of an early warning algorithm to detect sick broilers. Computers and Electronics in Agriculture, 144, 102-113. https://doi.org/10.1016/j.compag.2017.11.032

39. Zhuang, X., \& Zhang, T. (2019). Detection of sick broilers by digital image processing and deep learning. Biosystems $\quad$ Engineering, $179, \quad 106-116$. https://doi.org/10.1016/j.biosystemseng.2019.01.003 